\title{
Disability Management: Organizational Diversity and Dutch Employment Policy
}

\author{
Helen Kopnina • Joke A. Haafkens
}

Published online: 11 April 2010

(C) The Author(s) 2010. This article is published with open access at Springerlink.com

\begin{abstract}
Introduction While Human Resource Managers (HRM) and line managers could play a significant role in the prevention of job-related problems and in promotion of early job-continuation, it is not clear wether the chronically ill workers are recognized as a group. Unlike some other groups, distinguished by gender, age or ethnicity, those with chronic illness are less distinct and may not be included in diversity management programs. The aim of this research is to address theory and evidence in literature about the topic, as well as to inquire whether chronic illness of the employees is 'visible' in practice. Methods For desk research, we used a systematic search strategy involving medical, statistical, management, and social science databases (Web of Science, MedLine, Pub Med, Psych Info, etc.). Research results are based on case studies conducted with the managers and HRM of government and commercial organizations between March 2007 and October 2008 and between October 2008 and April 2009. These case studies were based on open interviews and focus group sessions (for human resource departments) which were consequently analyzed using thematical analysis. For group sessions, we used concept mapping to collect information from two groups of HRM professionals and managers. Secondary analysis included thematic and content analysis of 'best practice' organizations carried out by
\end{abstract}

\section{H. Kopnina ( $\square)$}

Amsterdam Institute of Advanced Labour Studies, University of Amsterdam, Plantage Muidengracht 12, 1018 TV Amsterdam, The Netherlands

e-mail: h.kopnina@uva.nl

\section{J. A. Haafkens}

Academic Medical Center, University of Amsterdam, Department of General Practice, Amsterdam, The Netherlands e-mail: j.a.haafkens@amc.uva.nl the Dutch organization Gatekeeper. Conclusions We have discovered that the chronically ill employees are largely invisible to HRM practitioners, line managers who do not always have the right instruments for implementation of the European or national frameworks. Most practitioners are unaware of the impact of chronic illness in their organizations and in employees work life.

Keywords Diversity - Disability management . HRM - Organizational policy · Chronically ill workers . Employment

\section{Introduction}

In recent years employees with a chronic illness have been increasingly identified as a specific group in EU and national policy documents. It has been argued that chronically ill employees should not only have as much access to the labor market as their healthy counterparts, but also that they should be able to retain their jobs if they have one. In the past in larger companies the task of guiding employees with (chronic) health problems was primarily designated to occupational health physicians or occupational health services. However, it is argued that Human Resource Managers (HRM) should play an active role in the guidance and management of chronically ill employees as part of diversity management policy [12].

Our research among employers and HRM aims to disclose the causes of invisibility of the chronically ill as a group and to deepen the understanding of the chronically ill employees as a distinct diversity group. The driving question of this research is whether chronic illness of the employees is 'visible' for line managers and human research managers in practice. We argue that the 
invisibility of chronically ill employees as a group may be explained by four main factors. First, by the fact that chronic illness is often literally invisible as its symptoms are often not manifest; second by the fact that chronically ill employees form an internally heterogeneous group; and thirdly by the fact that employers and HRM are not always trained to recognize such a condition. Finally, discrimination at the work place and stigma attached to chronic illness may inhibit disclosure.

Dutch employers are experiencing an increase in the number of chronically ill and disabled employees. This tendency has three main underlying reasons. First, improved medical technologies have reduced incapacitating morbidity and mortality among people with chronic diseases. Second, the percentage of the whole population with chronic illness increases due to the greying population. Third, due to recent policy shifts, Dutch disability benefits become harder to obtain and more chronically ill people seek or want to keep employment.

Dutch policies in regards to chronically ill employees are largely reflective of the European policy trends towards greater flexibility in the way of policy implementation as well as shared responsibility for successful reintegration between the employers and employees [12]. Recently, there has been a significant change in the Dutch policy concerning employment of disabled people, reflecting the need of the government to reduce the administrative burdens on companies and eliminate superfluous rules to give companies more scope for introducing individual arrangements. The new Working Conditions Act has been introduced on January 2007, under which both employers and employees have to cooperate with the return-to work plan. A return-to-work plan may include proposals from the employers for possible changes of the labour contract. The new act makes provisions for tailor-made rules by enabling employers and employees to consult each other before laying down agreements to ensure a healthier workplace. The new legislation acts in accordance with equal treatment and non-discrimination acts. The Labour Inspectorate will trust the agreements between employer and employee, but will take firmer action if the rules are abused [5].

\section{Definitions of Chronic Illness and Statistics}

One of the problems with identifying the chronically ill as a group is definitional-who is considered to be chronically ill or disabled? Most commonly used definitions stem from International Statistical Classification of Diseases and Related Health Problems (ICD) developed by World Health Organization (WHO) [6]. ICD distinguishes between common diseases and their associated biological and behavioral risk factors [6].
When defining a group of people as chronically ill, there are also social and cultural factors that play a significant role in how illness is experienced by sufferers and perceived by others. Here the accent lies on function of individual within society or at work, rather than on assumed objective manifestations of illness. The ICF classifies health-related domains from body, individual and societal perspectives [6]. The definition and the experience of chronic illness is thus far from fixed and is both culturally and contextually variable. Aside from the medical (technical) side of the disablement process, social, cultural psychological, and environmental factors also need to be taken into account [7].

This definitional difficulty is reflected in statistics, where categories of chronically ill or disabled may be blurred or overlap. By some estimates, chronically ill employees represent almost $40 \%$ of the entire workforce in the Netherlands [1]. In 2006, around $25 \%$ of population between the ages of 15-65 was classified as having a chronic illness and around $15 \%$ was work disabled. Among the working population these percentages were $19 \%$ and around $10 \%$, among the unemployed 28 and $17 \%$, and among the non-working the percentages are 37 and $28 \%$. A recent survey conducted by the Agency for Healthcare Research and Quality found that $48 \%$ of the population lives with some kind of chronic condition, and of these people, $60 \%$ of them work in some capacity [8]. Labour participation in The Netherlands increased from $63 \%$ in 2003 to $65 \%$ in 2007 . In 2008, 207,000 more people than in 2003 will have a job of 12 or more hours per week. An anticipated 310,000 people will receive social security benefits in 2007. That is approximately 20,000 less than in 2003. The number of benefits for occupational disability (including disabled youth) decreased sharply, from 980,000 at the end of 2003 to 825,000 in 2007. Some of the chronically ill are seeking work, others who do not seek work having gone through evaluation process by WAO, ${ }^{1}$ $\mathrm{WAZ}^{2}$ and $\mathrm{WAJONG}^{3}$; others are non-working (students, house-wives, etc.) [1]. One and the same person might be classified as belonging to one or more categories or changing category status throughout life duration (for example, from non-working student to the unemployed disability benefit recipient).

We may observe that the group of the chronically ill is very broad. This group, despite its heterogeneity, does have common characteristics. These include the duration of chronic illness (last longer than one year); physical or mental limitations; and the requirement of ongoing medical care. Symptoms vary in severity from mild to very serious

\footnotetext{
${ }^{1}$ WAO: Occupational Disability Act.

${ }^{2}$ WAZ: Incapacity Benefit for Self-employed.

${ }^{3}$ WAJONG: Incapacity Insurance for Young Disabled Persons.
} 
and do not always follow an expected pattern (flare ups followed by remission periods versus constant symptoms). Diseases also vary in symptom visibility and progression.

\section{Disability Within Diversity Management}

Diversity management refers to 'the systematic and planned commitment on the part of organizations to recruit and retain employees from diverse demographic backgrounds' [2]. Diversity management is concerned with acceptance of a multicultural workforce comprising employees with diverse ethnic, racial, religious and gender backgrounds. This diversity necessitates adopting appropriate strategies for its management [3]. While gender, ethnicity and age are widely discussed topics in the diversity management literature, chronically ill workers are largely invisible to the diversity management practitioners.

Within HRM, organizational diversity implies creating a company policy and managing human resources to reduce differences between employees and to use potential of different groups of workers [4]. Diversity managers were criticized for attempting to form a collective whole out of shifting and diffuse groups as well as trying to establish and fix the differences between the groups while homogenizing differences within the groups. Roosevelt Thomas Jr. [9, 10], a significant critical expert who addresses some of the potential dangers of affirmative action programs, criticized the definition of diversity: 'By limiting the term to minorities and women, and ignoring diversity's other dimensions, this definition blocks understanding. It is extremely difficult, for example, for a white male who defines diversity in terms of minorities and women to stifle concerns about reverse discrimination and preferential treatment' [10].

In their provocative article 'An Overlooked Dimension of Diversity: The Career Effects of Chronic Illness', Beatty and Joffe [8] argue that it is easy to overlook the hidden effects of illness or to interpret them as an individual rather than a collective concern, due to some flawed assumptions about human resource planning for this group of employees.

One assumption is that this niche population is irrelevant to organizational planners because people with chronic illness either will not enter the workforce or will leave soon after diagnosis. Another assumption is that the accommodation needs of people with chronic illness are fully met within the existing mechanisms used for other groups, such as people caring for small children or elderly parents, and therefore no special attention is necessary. Closely related to this is the assumption that chronic illness can be addressed the same way as acute illnesses, using primarily sick leave and temporary readjustments of work [8].
Too often organizations are unaware of the impact of chronic illness on an employee's work life. Employees generally leave an organization without asking for the additional flexibility that could have helped them stay at their jobs. Or, the situation only becomes apparent when the employee files a disability insurance claim, when it is too late for any intervention. Increasing organizational awareness of these issues will help to retain talented workers and allow people with chronic illness to continue to contribute to the organization.

Beatty and Joffe have explored the unique career patterns that characterize people living with chronic illnesses, suggesting that this group shares some elements of a common social identity. They argued that while sharing characteristics of other social identity categories, chronic illness has characteristics that distinguish it from other kinds of diversity: it is unpredictable in disease progression, variable in how it affects individuals, often invisible, and permanent. Beatty and Joffe concluded that chronic illness is a unique diversity category and that recognizing and understanding how these chronic illness characteristics affect an individual is a critical aspect of diversity awareness and essential for designing effective and compassionate accommodation strategies [8].

Chronic disease and disability management has occasionally been incorporated into diversity management but normally indirectly. Chronic Disease Management (CDM) is an approach to health care that emphasizes helping individuals maintain independence and keep as healthy as possible through prevention, early detection, and management of chronic conditions.

Many international examples of chronic disease management are known. In the Netherlands, disability management is defined as a corporate approach to enable organizations to take an active stance in maintenance and optimal functioning of employees with health problems. Increasing participation of employees with disability or handicap, employing workers with partial work disability and offering them a new chance at labor participation is outlined as one of the major goals of re-integration policies initiated by the Ministry of Social Affairs and Employment. These state initiatives are translated into organizational policies referred to either as prevention or labour conditions policy, sick leave policy or simply personnel policy.

Still, the reports of the Ministry of Social Affairs and Employment on disability management indicate that the policy in most organizations is implemented ad hoc. The supervision of the chronically ill employees often happens at the individual level, without researching the deeper underlying cause of such a fall-out and without comparison or consultation with other similar cases within the given organization. In the recent Dutch studies of opinions of the 
chronically ill employees it appears that Dutch employees are still rather reluctant to reverse negative stereotypes associated with illness and handicap, which could allow greater labor participation of the chronically ill. There is little awareness of the fact that the chronically ill represent a large group within the labour force and of specific requirements that this group might have.

\section{Case Studies}

In the qualitative study using 2 concept mapping sessions with 27 HRM professionals and managers, ${ }^{4}$ Haafkens [11] identified 6 themes. Common themes were: There is a need for "clear company policy", "employees who take their own responsibility", "more knowledge among HRM/ managers about chronic disease and it's prevalence in the company", "work adaptations". One theme was only mentioned by managers: "good cooperation between the manager and employee". Themes only mentioned by HRM professionals were: "a culture of trust, openness and communication within the organization" and "support within the organization".

This study revealed HRM professionals and managers are similar in some aspects and differ in other aspects with respect to what they perceive as important for enabling sustained employability of chronically ill employees in the work-place. Both groups perceive it as important that managers and HRM professionals have knowledge of chronic diseases and of the prevalence of these diseases among employees. A closely related issue is that the employees themselves must take action by disclosing their situation and their needs to their supervisors, personnel officers, and colleagues. Two other common themes were "the provision work adaptations by the organization" and the "development of a company policy that allows for attention to the needs of the chronically ill employees and the company".

Differences were that managers saw "good cooperation between the managers and chronically ill employees" as a specific condition. HRM professionals created two distinct themes: a "company culture that allows for trust, openness and communication between workers and management" and "a supportive organization". These variations may not be surprising, because managers and HRM professionals have different tasks within the organization and therefore different interpretative frameworks to address problems.

\footnotetext{
${ }^{4}$ Concept mapping is a structured methodology for eliciting, organizing and aggregating the ideas of groups of diverse stakeholders on a certain focus question. It uses qualitatively collected data from group members and results in quantitatively derived graphic maps (concept maps) displaying the interrelationships among ideas expressed by the group and its sub-groups.
}

The former have closer and more frequent contact with employees. For that reason they may put more weight on the nature of the relationship between management and employee. The latter have a more distal relationship to individual employees, but a wider view of company values regarding worker's health and development. For that reason they may put a greater emphasis on what an organization may do to facilitate cooperation in terms of its policy and its culture [11].

There are few best practice examples of disability management available in The Netherlands. The Gatekeeper, the employer forum that supports companies and institutions incorporating health management in their company culture, distinguishes between the 'good practice' organizations on the basis of their success in employing, supporting and re-integrating chronically ill employees. These good practices can be characterized by realization of the diversity within its own organization with the goal of increasing participation of employees.

Aside from these studies and reports, very little is known about disability management as part of diversity management within the HR or employment policy in The Netherlands. Even less is known about how employers' and HRM's opinions are translated into policy-implementation actions. We shall hereby examine four case studies of managers and HRM within five Dutch organizations, conducted between October 2008 and April 2009.

\section{University of Applied Science (HS)}

University of Applied Science (HS) has some 1,600 staff (roughly half of which work full-time) and about 17,000 students. The central policy is formulated through the government the general regulations for civil service workers. As most organizations in The Netherlands, HS formulates the equal opportunities policy. This policy implies that all employees and job applicants are treated fairly and equally, regardless of their sex, sexual orientation, marital status, race, colour, nationality, ethnic or national origin, religion, age or disability.

During interviews with HR representatives $(n=8)$ and line managers at HS $(n=4)$, it emerged that it is normally the HR representative who get notified about a health condition by chronically ill workers. This happens in cases when the employee tries to avoid disclosure with the line manager, yet illness is perceived by the employee as interfering with his work tasks. HRM department of HS does not normally disseminate this information to the line managers. Information is sometimes conveyed to the top management, if redeemed important and with the employee's consent. Sometimes HRM receives information from the corporate doctor and then either contacts the employee or in some cases both employee and the line 
manager. Depending on a number of factors, such as the employees' wishes, severity of reported disorder, degree of interference with and the ability to perform the work task, cooperation of the line manager plans of action are formulated.

Eventual decisions in regard to these cases (possibility of making structural or functional adjustments, termination of employment, etc.) are made and implemented by either the line managers in consultation with the employee and HRM, or by the line manager in consultation with the top manager, or simply between the HRM and the employee (in case no major adjustments are needed). One of the HRM representatives summarized this process as being 'somewhat arbitrary' and 'depending on individual case'. Subjectivity of such a decision making process is confirmed by one of the line managers, a middle-aged male head of one of the academic departments:

Sometimes we 'follow the book'. But the book does not spell out what needs to be done in each individual case. We know we cannot discriminate and we need to support chronically ill workers. But $[\ldots]$ how it really happens at the grass root level is a different question. Sometimes it all depends on employee's disclosure. Sometimes it's the boss who needs to make the first step... noticing that someone [employee] isn't performing well... [Interview April 2008, translated from Dutch by author H. K.]. ${ }^{5}$

The same respondent reflected on the question what he considers to exemplify 'best practice' or 'good strategy':

There might be as many opinions about it as there are people in the room [8]. I don't personally think there is one winning formula or an ideal prescription for what needs to be done. Maybe sensitivity to each individual case is the most important guiding principle... I would however suppose, leaning upon my previous experiences, that a centralized system in which it's the [top] management that makes all the decisions [regarding chronically ill employees] isn't that effective. After all, these [chronically ill] employees are not all the same. You can not require a person to talk about their condition, let alone treat it in accordance to what a [corporate?] doctor or the boss says. If you threaten [the employee] with firing them if they miss a lot of [work] days-that's not an effective strategy to promote disclosure and deal with the condition... There needs to be a dialogue to discuss what the possibilities are, what special needs need to be met or what expectations are...

\footnotetext{
5 All quotes in this article are from interviews conducted between April and June 2008, translated from Dutch by the author.
}

A female member of HR department disagrees:

It's easy to say: let the worker come to us. What if they don't? And how are we supposed to deal with it? HRM are not specialized [medical] professionals. If we are not asked to convey the information further [to the employer], we are left with the question of what to do about it... It's one thing getting a complaint about health and it's another question of whether HRM should be a bridge between the worker and the management. This could also lead to having to choose sides [employee or employer]... Ideally, I think, there should be strict policy rules according to which worker should feel protected enough to talk to his boss directly.

It appears that interpretation and implementation of policy related to formal sickness prevention and absenteeism is largely left to the stakeholders within the organization itself. The process of information acquisition and dissemination within this organization is rather sporadic and decentralized.

\section{Super}

Another case is that of Super, a Dutch supermarket chain store. At the chosen location Super has the team leaders for the different areas, who are responsible for groups of 20-30 employees, such as the controllers and the secretaries. There are some 619 Super employees; 55\% full-time and about $45 \%$ temporary personnel.

The interview was recorded with Martijn D., the line manager, and Frank T., the physiotherapist from Tigra Rotterdam, a training institute for health, recreation and work. Physiotherapists are hired in at Super to rate and treat employees if they have reported physical problems. This is done in close cooperation with the company's medical officer. Frank has consultation hours three times a week and the team leaders can make an appointment for an employee, either preventively or in the case the employee already has physical discomfort.

According to Frank, the corporate doctor's office is normally the place where the chronically ill go to for advice: 'They can also go to the team leader, and then they can make an appointment with me or the company's medical officer'. Martijn compliments this by saying:

If there is a problem with things such as back or shoulder they can easily make an appointment with Frank, thus it is easy to plan. For other things, such as problems with lungs, they can go to the doctor within the company. Many companies do not have that possibility for employees, as they do not have a doctor and physiotherapist within the company... 
Reflecting on the Dutch policies regarding chronically ill, Martijn says: 'Well, years ago we had medical tests for people that applied for a job. Nowadays this is not the case anymore, but of course it makes a big difference. It is now only for certain jobs, where it is really necessary'. According to Martijn, Dutch and other European policies greatly differ:

There are differences within Europe; Germany is different from the Netherlands... If you are disabled or have discomfort here in Holland, you have to go to an institute called UWV, this institute judges if you are disabled or not... If you have some kind of disability in your back, you could still do some administrative work for example. Therefore you do get some subsidies from the government... If, on the other side, you have something very serious, such as cancer, you will get the whole amount of the aid offered by the government. But the Dutch government is becoming stricter with giving out those aids.

For individual organizations, reflects Frank, different rules may apply, particularly including the possibilities for subsidies: "In the Netherlands the government subsidizes companies that employ those people. Thus they make it appealing to firms to employ chronically ill or disabled people. Those subsidies can be money, but also other things...." Martijn adds: "it can be a bit scary for the employee to employ a chronically ill person, especially when you don't know how it is with subsidies and such, as there is always a bit more risk involved when employing someone with an illness'.

Unlike in the case of HS, the line manager and the physiotherapist reported that they receive information about the chronically ill employees regularly, and that there is an established procedure for treating different illness cases.

\section{C-a}

$\mathrm{C}$-a is part of an international company, which specializes in innovation in the area of raw material use. The company has approximately 4,000 employees, working in 36 countries. C-a formulates the so-called whistle blowing policy which allows employees to raise concerns with management about the conduct of others which they consider to be in some way damaging to the organization or others within it. C-a also formulates the equal opportunities policy and has some special internal policies concerning the chronically ill workers.

Nel Van G., policy advisor and an occupational health nurse who works in C-a's HRM department stated that chronically illness is not of an importance for $\mathrm{C}-\mathrm{a}$, the employees are never discriminated by race, religion, gender, age or health. Nel also stated that although the company has a low proportion of chronically ill workers (3.1\%), the management and HR Department try to build respectful and beneficial relationships between the managers and the workers. For instance, people with diabetes to have a day-night rhythm otherwise they can get sick, so $\mathrm{C}$-a offers them a special working schedule with no night shift. C-a also makes effort to improve working conditions for chronically ill workers, for example change their work place, and provide special equipment such as chairs.

Through contacting Nel, sick employees they can get necessary help from the company doctor. Chronically ill people can enjoy certain tax reduction through pensions or tax benefits, if they stop working. If there are a lot of chronically ill people, Nel states that it is also possible to get tax reductions and some financial discount from the company. At the age of 55 and older, people that can not keep up with their function because of a chronic sickness can get transferred to a lower job position in the company but still get paid the same amount of salary as before. The procedure for disclosure and adjustment may vary but generally Nel feels that $\mathrm{C}$-a is open to anybody requesting special equipment or adjustment:

He or she does not have to say that he or she is sick and what kind of sickness he or she has. There is no difference for our company whether a worker is sick or not because if he is, then he gets special work environment in order to help him feel better at work. $\mathrm{He}$ can get special furniture or work different times than normal schedule...

Yet some functions within $\mathrm{C}$-a require worker testing having to do with their specialization- Nel particularly refers to manual factory workers.

Some... chronically ill workers... can be a very suitable candidates for a certain work placement... They can start or continue working and if their health condition becomes worse, the company can always help him with it. For example, if someone has diabetes and their condition becomes worse, they can work fewer hours than before to improve their health... Some of the things that can be changed are: working hours, space on their work place, arranging special furniture... [Eventually the worker may be] transferred to a lower job position but still get paid the same amount of salary.

$\mathrm{Nel}$ feels that communication between the ill worker and the manager in $\mathrm{C}$-a is well arranged. Nel feels that clarity and simplicity (of approach) is necessary and that it helps that she is both the contact person and the company doctor. 
Et./Ad.

Ad. is an international group of quality supermarkets based in the United States and Europe. Acquired by Ad. in 1974, Et. is the leading drugstore, health and beauty-care retailer in The Netherlands. We have interviewed the Human Resource manager of one of the Et. locations Gerard K. (location and details undisclosed). Gerard reflects that there are large issues associated with illnesses of the employees particularly because of the physical nature of work (mostly standing and walking). Gerard thinks that the chronically ill work mostly in the headquarters in administrative jobs and that "percentage of the chronically ill people in the shops is zero because they can't work there". While there are more chronically ill working at the shops, Gerard reflects, the only really suitable working place is in the headquarters since there are more administrative jobs available. Corporate doctor may evaluate the working possibilities and adjustments for an individual worker.

At the location in Rotterdam Gerard spoke once to a chronically ill lady with reuma who was persistent in trying to work at the shop despite her disability:

She can't give money back to the customer... She can't put a shampoo on the shelf. It is impossible for her. So I told her we are going to a reintegration office to look for her to get a job somewhere else, outside of the Et... But we always help people if they are ill, and it is not working at the Et., to find work elsewhere. And we pay for it... When someone is ill for two years we pay them salary... After the first year we look, okay, can this person come back in the labor market. Well this lady with reuma is not coming back. We also got that checked by an agency. And the coming year the reintegration office is going to look how they can educate her to a job somewhere else.

Nonetheless, Gerard states, Et. does not try to discriminate against workers or potential applicants. Still, hiring procedure necessitates some disclosure in older applicants:

Sometimes [our applicants] have a WAJONG benefit... Young people that get a WO benefit, they can also send an application to us... But every employee that is coming to us when he is older then 22, they get a letter from us; do you have a WAJONG benefit, yes or no. When someone has a WAO benefit, we get a lot of money back from the government.

According to Gerard, Et. does not have a consistent policy, while Ad. does. There is some sporadic documentation, as in recording the numbers of chronically ill employees and the nature of their illness. The data is sometimes entered into the company's data base but managers or HR representatives at other Et. locations, but Gerard does not know what happens to it afterwards and weather the data is consistent with the actual numbers of the chronically ill. When asked whether he is satisfied with the existing policy at Et., Gerard replied: "At the headquarters, I think yes. In the distribution centers and the shops, forget it".

$\mathrm{N}-\mathrm{a}$.

$\mathrm{N}$-a. is a supermarket based in Nieuwegein, focusing more on offering the lowest price guarantee on it is products and less on the customer service. $\mathrm{N}$-a. has 74 employees, twothird of which works part-time. Most of them are students and housewives. According to the interviewed manager, two of the employees are chronically ill at the moment. The manager interviewed, T., has almost 25 years of experience in the supermarket business, 8 of them working as a manager at the $\mathrm{N}$-a. He considers the work load in a supermarket relatively high compared to a job in an office.

Workers have a heavy work load moving and lifting boxes and products and sitting for $8 \mathrm{~h}$ scanning the product at the cashier. Many people that work fulltime get sick because of this. Especially the cashiers they complain allot about sitting for many hours straight... They get complaints such as back pain and their legs. Also headaches occur because of the scanning beep...

There are set procedures to follow in case of sudden illness, but not necessarily in case of chronic complaints. When an employee gets sick he or she has to call early in the morning to inform the manager about his condition. The manager reports this employee's sickness to the head office trough the intranet. When it is a temporary illness such as a cold or fever they mostly take a few days to a week sick leave and call in when they are better. When it is a serious condition the procedure is the same as for temporary illness.

But when the employee is not back to work within 8 weeks the head office of $\mathrm{N}$-a. sends Commit Arbo [Corporate doctors' organization].. in order to get a health checkup. Commit Arbo helps employer and employee find a way to remain the employee's job... Disabled or elderly people cannot work in a supermarket because of the heavy work load. The $\mathrm{N}-\mathrm{a}$. has no time and money to set up special equipment for the needs of those people.

During the application procedure managers screen the applicants through oral questioning and a question in the application form. T. also checks formal employers and asks for references. A supermarket does not have many 
employees that are not easy to replace. Everyday new floor workers and cashiers apply for a job. Part-time employees that apply get a temporary working contract which is automatically renewed every 6 months three times. After the third time the employee does not get a new contract anymore. Part-time employees do not get paid when they are sick.

T. considers the supermarket too small to have a policy towards the chronically ill nor to have any trainings or courses for managers or employees. To illustrate his own experience with the chronically ill $\mathrm{T}$. gave an example of a young man with back pain. He has been working the whole year off and on. So the N-a. contacted Commit Arbo and scheduled a meeting between a Commit Arbo representative, the $\mathrm{N}-\mathrm{a}$. Manager and the worker.

They have agreed that the worker has to do exercise physical therapy or go to a center for sport and physical activities. This worker used to work $40 \mathrm{~h}$ a week. Now in order to retain his job again he works for 2-4 $\mathrm{h}$ a day. Once he tried to work for $6 \mathrm{~h}$ in a day but that was too much for him...This disclosure is based on trust.

$\mathrm{T}$ reflects that the $\mathrm{N}-\mathrm{a}$. has to be more socially sensitive. A confidential person that the employees could talk to when they have problems could be one solution. Also house visits from the manager once a week to chronically ill workers could help. $\mathrm{T}$ thinks the most important for the ideal policy for the chronically ill is to assume control of the illness of your employees. There should be an information center within the organization specializing in chronic diseases and the HR representative should know which employees have which illness. Equally important is that a company doctor knows about all employees' conditions. It is also important that employees and managers should have trust and good relations with each other and that manager should listen to the problems of employees and help them with solving them without the need of disclosure to colleagues.

\section{Discussion}

Reflecting upon literature study, we notice that the chronically ill differ from other diversity groups, characterized by socio-demographic factors such as ethnic descent, age and gender by a number of factors:

1. Chronically ill as a group are very diverse and crosscut other categories like age, gender and ethnicity.

2. Symptoms and work-related abilities vary greatly.

3. In most cases chronic illness is not visible.

4. Disclosure is difficult due to stigma and other social factors.
We also notice that there is a large amount of flexibility possible within different organizations as far as interpretation of policy is concerned. Policies are interpreted and implemented in accordance to specific organizational structure and culture rather than following general guidelines. We may place organizations in a few categories according to relationships and hierarchies between departments and stakeholders' groups such as line managers, top managers, HRM or other stakeholders. These categories relate to the way stakeholders:

1. formulate policy

2. interpret policy

3. implement policy

4. receive, control and disseminate policy-relevant information

5. implement eventually adjusted policy

Reflecting upon the case studies, we notice that there is a large amount of flexibility possible within different organizations as far as interpretation of policy is concerned. We may conclude that policies are interpreted and implemented in accordance to specific organizational structure and culture rather than following general guidelines. This reflects the general trend within European and Dutch policies in allowing organizations greater flexibility in employee-employer relations.

\section{Conclusion}

We have discovered that the topic of the chronically ill employees is largely invisible to HRM practitioners, line managers and diversity specialists who do not always have the right instruments for implementation of the European or national frameworks. Line managers and human resource managers use their personal opinions for handling the chronically ill employees. Most practitioners are unaware of the impact of chronic illness in their organizations and in employees work life.

Organizations vary in accordance with particular arrangements in the way information about the chronically ill is disseminated, processed and how the decisions are made. When our database is expanded with a wider set of case studies, we may be able to classify the organizations in accordance to different institutional or cultural characteristics and deduce which arrangements work better in enabling the chronically ill employee to continue optimally functioning at work.

Returning to the faulty assumptions about the chronically ill by Beatty and Joffe [8], we recall that the managers often assume that the chronically ill will not enter the workforce or retire soon after the diagnosis. Yet, as Beatty' and Joffy's own study as well as some the aforementioned 
studies show, people experiencing disabling symptoms often continue to work. Beatty and Joffe give two reasons for this. First, a critical distinction is whether the disabling symptom interferes with job skills essential for performance. Second, recent improvements in medication allow people to manage disabling symptoms more effectively so effects on performance can be minimized. It is true that people with chronic illness benefit from flexible schedules and periods of sick leave. However, both these assumptions fail to recognize the unique features of chronic illness that call for other kinds of accommodation in job design [8].

We have established that the group of the chronically ill is very broad. Heterogeneity of this group as well as social factors such as prevalence of stigma as well as the fact that employees are rarely trained to recognize chronic illness account in large part for the group's invisibility.

However, the group does have some common characteristics, including the long duration of chronic illness; and physical or mental limitations. There are also common needs that most group members can identify with, such as requirement of ongoing medical care, the need for recognition and acceptance, and often the need for more flexible, open and humane organizational culture. Approaching the chronically ill employees as a group may be constructive in both serving the employers' need to retain good workers and the employees' desire to retain job and the need to be treated equally.

Open Access This article is distributed under the terms of the Creative Commons Attribution Noncommercial License which permits any noncommercial use, distribution, and reproduction in any medium, provided the original author(s) and source are credited.

\section{References}

1. Central Bureau of Statistics, The Netherlands, CBS; 2008.

2. Thomas R. From affirmative action to affirming diversity. Harvard Business Rev. 1990;68(2):107-17.

3. Edawor PA, Aluko YA. Health and employers' responsibility. Int J Divers Org Commun Nations. 2009;6(6):189-96.

4. Disability management forum http://www.ifdm.com.au/docs/ IFDM2006-abstracts.pdf.

5. European Agency for Safety and Health at Work. The New Dutch Working Conditions Act. http://nl.osha.europa.eu/legislation/ ?language $=$ en.

6. World Health Organization (WHO) (2010). ICD http://www.who. int/classifications/apps/icd/icd10online/.

7. Verbrugge L, Jettie AM. The disablement process. Soc Sci Med. 1994;38(1):1-14.

8. Beatty JE, Joffe R. An overlooked dimension of diversity: the career effects of chronic illness. Org Dyn. 2006;35(2):182-95.

9. Thomas RR Jr. Beyond race and gender. New York: Amacon; 1990.

10. Thomas Jr RR. A diversity framework. In: Chemers MM, Oskamp S, Constanzo MA, editors. Diversity in organizations. New perspectives of a changing workplace. Thousand Oaks, London; 1995. pp. 245-263.

11. Haafkens J. Perspectives of HRM professionals and managers on what policies and practices are needed within an organization to enable sustained employability for chronically ill employees. Working paper, 9th international conference on human resource development research and practice across Europe: Lille; 2008. pp. 21-23.

12. De Smedt M, Van den Berg JK. Indicators disability and social participation in Europe. Brussels: European Commission Eurostat; 2001. p. 30. 